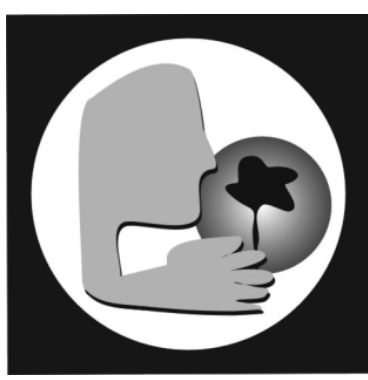

Sustentabilidade e $m$ Debate

\title{
LACIS/FAU/CDS/FGA-UnB: Inovação e Sustentabilidade
}

\author{
Raquel Naves Blumenschein ${ }^{1}$, Maria Vitória Ferrari Tomé2
}

1. Doutora pela Universidade de Brasília, Professora Adjunta da Faculdade de Arquitetura e Urbanismo da Universidade de Brasília e Coordenadora do Laboratório do Ambiente Construído, Inclusão e Sustentabilidade (LACIS-

CDS/FAU-UnB). raquelblum@terra.com.br

2. Doutora pela Universidade Federal de Viçosa, Professora Adjunta da Faculdade UnB Gama, Vice-Coordenadora do Laboratório do Ambiente Construído, Inclusão e Sustentabilidade (LACIS-CDS/FAU-UnB). vitferrari@gmail.com

Recebido em 20.03.2011

Aceito em 30.05.2011

\section{RESUMO}

Palavras-chave: inovação, pesquisa aplicada, redes colaborativas, cadeia produtiva da indústria da construção, gestão de resíduos sólidos, processos regenerativos urbanos e sociais.
Os desafios do século XXI exigem que mudanças de paradigmas tecnológicos sejam aceleradas visando minimizar impactos ambientais e desperdícios de recursos naturais, e reduzir emissões e poluições. Entre as atividades mais impactantes no planeta cita-se a produção do ambiente construído que é resultado de processos da Cadeia Produtiva da Indústria da Construção (CPIC). O estudo da aceleração da mudança de paradigmas tecnológicos está vinculado ao estudo do processo de inovação. A compreensão dos vetores de influência no processo de inovação da CPIC requer estudos que identifiquem como novas idéias são geradas e integradas ao processo de produção e aos produtos, o que exige que modelos específicos sejam desenvolvidos e testados na prática por meio de projetos pilotos. Os estudos que visam identificar as forças responsáveis pelo surgimento das inovações e que demonstram, com mais profundidade, as relações entre os diferentes atores e os vetores de influência se tornam relevantes. Neste sentido, entre as contribuições que vêm sendo dadas no âmbito da Universidade Brasileira, cita-se o Laboratório do Ambiente Construído, Inclusão e Sustentabilidade (LACIS/UnB). O objetivo deste artigo é apresentar a trajetória do LACIS/ FAU/CDS/FGA-UnB nos últimos cinco anos e os principais resultados da pesquisa aplicada à criação, teste e compartilhamento de tecnologias, metodologias e conhecimento com foco na cadeia produtiva da indústria da construção, gestão integrada de resíduos sólidos e processos regenerativos sociais e urbanos. A apresentação do processo evolutivo do LACIS requer a demonstração dos princípios teóricos que fundamentam a sua evolução, as origens e etapas do seu desenvolvimento e os resultados dos projetos de pesquisa aplicada.

\section{ABSTRACT}

The twenty-first century challenges require the acceleration of the technological paradigms changes in order to minimize environmental impacts and waste of natural resources, and reduce emissions and pollution. Among the main impactful activities on the planet quotes the production of the built environment which is a result of the processes of the productive chain of the construction industry (PCCI). The study of the acceleration of technological paradigm shift is linked to the study of the innovation process. The understanding of the influence vectors of the innovation process of PCCI requires studies that identify how new ideas are generated and integrated into the production process and products. These studies require the development of specific models which must be tested in practice through pilot projects. Studies aimed at identifying forces responsible for the emergence of innovations and demonstrating the relations between different actors and vectors of influence become relevant. In this sense, among the contributions that are being given in the University of Brasilia it can be quoted the Lacis/UnB. The aim of this paper is to present the trajectory of Lacis/FAU/CSD/FGA-UNB in the last five years and the main results of applied research with a focus on the productive chain of the construction industry, integrated management of solid waste and regenerative urban and social processes. A presentation of the Lacis evolutionary process requires the demonstration of theoretical principles that underlie its evolution, the origins and stages of its development and the results of the applied research projects. 


\section{Introdução}

Os desafios do século XXI exigem que mudanças de paradigmas tecnológicos sejam aceleradas visando aumentar a capacidade de sobrevivência de ecossistemas, minimizar impactos ambientais e desperdícios de recursos naturais e reduzir emissões e poluições. Entre as atividades mais impactantes no planeta cita-se a produção do ambiente construído que é resultado de processos da Cadeia Produtiva da Indústria da Construção (CPIC).

O estudo da aceleração da mudança de paradigmas tecnológicos está vinculado ao estudo do processo de inovação. A compreensão dos vetores de influência no processo de inovação da $\mathrm{CPIC}$ requer estudos que identifiquem como as novas idéias são geradas e são integradas ao processo de produção e aos produtos, o que exige que modelos específicos sejam desenvolvidos e testados na prática por meio de projetos pilotos.

Os estudos que visam identificar as forças responsáveis pelo surgimento das inovações e que demonstram com mais profundidade as relações estabelecidas entre os diferentes atores e os vetores de influência se tornam relevantes. Neste sentido, entre as contribuições que vêm sendo dadas no âmbito da Universidade Brasileira, citase o LACIS/UnB.

A criação do Laboratório do Ambiente Construído, Inclusão e Sustentabilidade (LACIS) é resultado de pesquisas de doutorado no Centro de Desenvolvimento Sustentável (CDS/UnB) e foi concebido em 2004, como uma parceria entre o CDS/UnB e a Faculdade de Arquitetura e Urbanismo (FAU/UnB). Em 2010, na ampliação e reestruturação do LACIS, a Faculdade UnB Gama (FGA) passou a ser parceira.

O LACIS visa à pesquisa, ensino e extensão com o foco em inovação e sustentabilidade do ambiente construído, considerando as redes colaborativas, a inclusão social, a educação ambiental e o fortalecimento do sistema de aprendizado de indústrias e de outras organizações.

A visão do LACIS preconiza a pesquisa aplicada que integra responsabilidade, recursos e soluções tecnológicas como vetor de inovação e transformação para contribuir para a regeneração da sociedade. E assume como missão a criação, teste e compartilhamento de tecnologias, metodologias e conhecimento com foco na cadeia produtiva da indústria da construção, gestão integrada de resíduos sólidos e processos regenerativos sociais e urbanos, que constituem os três eixos estratégicos de pesquisa do LACIS.

O objetivo deste artigo é apresentar a trajetória do LACIS/FAU/CDS/FGA-UnB nos últimos cinco anos e os seus principais resultados.

A apresentação do processo evolutivo do LACIS requer a demonstração dos princípios teóricos que fundamentam a sua evolução, as origens e etapas do seu desenvolvimento e os resultados dos projetos de pesquisa aplicada.

\section{Princípios Teóricos}

Três princípios teóricos são relevantes para a fundamentação e desenvolvimento deste artigo: o Sistema Nacional de Aprendizado (Viotti, 2000), fundamentado na teoria da inovação; a teoria de sistemas aplicada ao estudo de sistemas organizacionais, de acordo com os princípios da biologia (Bertalanffy, 2010; Capra, 1996, 2000); e a história natural da inovação, (Johnson, 2010).

\subsection{Teoria da Inovação}

Schumpeter (1949) definiu inovação como o desenvolvimento de novas combinações de fatores de produção. O surgimento descontínuo 
dessas novas combinações, ou o emprego de recursos existentes de diferentes maneiras, caracterizou o fenômeno do desenvolvimento econômico. O conceito de desenvolvimento de novas combinações inclui: “(a) a introdução de um novo produto; (b) a introdução de um novo método de produção; (c) a abertura de um novo mercado; (d) a conquista de uma nova fonte de fornecimento de materiais ou produtos manufaturados; (e) o desenvolvimento de novas formas de organização de qualquer indústria, como por exemplo, a queda ou criação de uma posição de monopólio" (Schumpeter, 1939:66).

A teoria econômica tradicional demonstra como elementos e partes do sistema econômico buscam um estado de equilíbrio que por alguma razão (por exemplo, alteração por demanda ou por fornecimento) pode mudar, causando uma reação desses elementos e partes, trazendo o sistema de volta a posição inicial. Schumpeter (1949) discordou desta abordagem estática, que assume mudança como adaptativa e responsiva, sempre buscando uma posição de equilíbrio. $\mathrm{Na}$ busca por uma explicação para o processo de evolução do sistema capitalista, o autor forneceu uma abordagem dinâmica para a compreensão de como o sistema econômico funciona e evolui.

O fenômeno chamado 'desenvolvimento' era entendido por ele como a alteração na vida econômica, que não é uma função de um fator externo, mas surge de dentro por sua própria iniciativa (SCHUMPETER, 1949:63). Apesar do reconhecimento da relevância dos fatores externos como variação de demanda, e de fatores internos como crescimento, que podem interagir e causar mudanças no sistema econômico, a inovação é um fator interno do sistema econômico, e tem um papel preponderante: inovação é um fator excepcional na história econômica da sociedade capitalista (Schumpeter, 1939, vol. I: 86).

Os esforços realizados por países em desenvolvimento para completar suas estruturas produtivas são importantes para o crescimento do país. Por outro lado, o crescimento econômico está ligado à capacidade de diversificação industrial associando transferência e difusão de tecnologia. Isso leva à tendência de que os países ao absorverem tecnologia, fortaleçam suas capacidades em introduzir melhorias incrementais contínuas aos seus processos de produção e produtos, aumentando suas oportunidades de crescimento.

Para que haja um ambiente favorável à mudança tecnológica é necessário que as capacidades tecnológicas tenham sido desenvolvidas paralelamente à capacidade de produção (Bell, 1993 apud. Viotti, 1997). Em países com industrialização tardia não se verifica um padrão seqüencial nas cadeias de produção, o que ocasiona um descompasso na sequiência de desenvolvimento tecnológico e a formação de uma estrutura industrial incompleta. Em um primeiro momento há a introdução de tecnologia de produção em produtos de consumo e mais tarde introduz-se tecnologia em elos iniciais da cadeia, por exemplo, (Viotti, 1997:84). Outro ponto neste contexto é que ao se introduzir uma nova tecnologia, a mão-de-obra pode não estar preparada para sua aplicação.

Essas deficiências foram identificadas por Viotti (1997) como características ligadas e dependentes de Sistemas Nacionais de Aprendizado (SNA). O sistema nacional de aprendizado tem suas raízes no conceito de Sistema Nacional de Inovação (SNI), definido como "a rede de instituições no setor público e privado cujas atividades e interações iniciam, importam, modificam ou difundem inovações" (Freeman, Apud. Viotti,, 1997:23). 
Entre as deficiências do SNA de países e indústrias com descompasso de inovação citamse: industrialização tardia, mudança tecnológica limitada ao aprendizado, estrutura industrial incompleta e mudança tecnológica dependente de crescimento. A diferença entre inovação radical e inovação incremental passa então a ser relevante nesta análise, sendo "seu padrão de difusão" (Freeman, 2000:467).

Inovações que envolvem mudanças na organização de produção e mercados são definidas como inovação radical, (Freeman 1988) implicando primeiro na introdução de um novo produto, processo ou forma de organização "inteiramente nova" (Lemos, 2000:158) e segundo, na mudança do paradigma tecnológico (Lemos, 2000 e Dosi, 1953, 1988).

A inovação incremental refere-se à introdução de melhorias em produtos, processos ou organização da produção, sem ocasionar mudanças industriais (Freeman, 1988) e está diretamente ligada à capacidade de aprender.

Viotti (1997) argumenta que há dois pólos nos quais se encaixam as estratégias de aprendizado de países com industrialização tardia: a estratégia de aprendizado passivo e a estratégia de aprendizado ativo. $\mathrm{O}$ primeiro conceito referese ao esforço tecnológico desenvolvido, que visa essencialmente à absorção da capacidade de produção, o que implica em dependência do crescimento econômico e do desenvolvimento de um padrão seqüencial de industrialização (Hirschman apud. Viotti,1997:112). O foco está em aprender o como produzir e está ligado apenas à difusão de tecnologia (Sistema Nacional de Aprendizado Passivo).

O segundo conceito refere-se ao esforço tecnológico desenvolvido, visando associar controle e conhecimento da produção a um esforço deliberado da melhoria da tecnologia de produ- ção empregada (Viotti, 1997:114). O foco passa a ser não apenas o como produzir, mas também como melhorar o que é produzido e o desempenho do processo de produção, ligado, portanto à inovação incremental (Sistema Nacional de Aprendizado Ativo).

A aplicação do conceito de Sistema Nacional de Aprendizado no lugar de Sistema Nacional de Inovação passa a ser mais adequada, segundo Viotti (1997), particularmente quando o foco é difusão e mudanças incrementais.

A utilização do conceito de SNA em estudos comparativos de países e cadeias produtivas é uma importante ferramenta. A dificuldade na disponibilidade de dados, no entanto, leva à utilização de indicadores macros (VIOTTI, 1997:180):

1. O nível nacional de educação e treinamento - depende da alfabetização e nível de escolaridade, número de engenheiros formados, treinamento em países estrangeiros, gastos públicos em educação (parcela do PIB) e qualidade da educação.

2. A aquisição de tecnologia - investimento estrangeiro direto, importação de bens de capital, compra formal e direta de tecnologia.

3. Os recursos nacionais comprometidos com aprendizado tecnológico - investimento do Estado em desenvolvimento de tecnologia.

4. Os resultados do esforço tecnológico nacional - eficácia dos esforços nacionais em disseminar determinadas tecnologias.

O fortalecimento do sistema de aprendizado é, portanto, um vetor relevante no processo de mudança de paradigmas tecnológicos. A sua aplicação no contexto da cadeia produtiva da indústria da construção (CPIC) ${ }^{1}$ tem permitido estruturar e nortear projetos de pesquisa aplicada que visam acelerar a absorção de paradigmas tecnológicos sustentáveis ${ }^{2}$ fundamentados nos 
princípios da conservação. Esses princípios defendem a manutenção dos principais processos ecológicos e dos sistemas de suporte à vida; a preservação da diversidade genética e a necessidade de assegurar a utilização sustentável das espécies e dos ecossistemas (Nature Conservation Act, 1992).

\section{2. Teoria de sistemas aplicada às organizações sociais}

Ao longo das últimas décadas, o desenvolvimento da teoria dos sistemas vem oferecendo uma base de integração do conhecimento científico em um amplo espectro: físico, biológico, social. A base inicial da teoria dos sistemas, em termos gerais, foi desenvolvida pelo biologista Bertalanffy, que previu que "na ciência moderna, interações dinâmicas é um problema básico em todos os campos, e seus princípios gerais deverão ser formulados com base na Teoria de Sistemas" (Bertalanffy, 2010).

A teoria organizacional tem buscado uma abordagem similar ao adotar o conceito de Sistemas Complexos Adaptativos (SCA), fundamentado e proposto a partir da teoria de sistemas, no qual o fator complexidade passa a ser inerente às organizações sociais. A visão de sistemas abertos foi introduzida nas organizações nos anos 1960 e a partir de então a complexidade se transformou em um constructo central no vocabulário dos cientistas organizacionais (Silva e Rebelo, 2003. Os sistemas abertos são assim denominados porque trocam recursos com o ambiente $\mathrm{e}$ são constituídos por elementos interconectados que trabalham juntos (SILVA E REBELO, 2003:782).

Os avanços da genética e da microbiologia têm contribuído para a compreensão da evolução dos seres vivos, a partir de uma mudança conceitual da teoria da evolução e dos sistemas vivos, que por sua vez tem reflexos em sua aplicação para o estudo dos sistemas sociais.

No discurso do Prêmio Nobel de 1983, a geneticista Bárbara McClintock explicitou este conceito refletindo sobre o futuro, que hoje é realidade, quando as atenções se voltaram para o genoma "um órgão altamente sensível da célula, que acompanha as atividades genômicas, corrige os erros comuns, percebe os acontecimentos estranhos e inesperados e reage a eles" (Bárbara McClintock, Apud. Capra, 2002:178).

A evolução passa a ser vista como um processo natural da vida e faz parte de sua autoorganização, sendo, portanto cognitiva, já que ao perceber erros ou acontecimentos incomuns reage a eles, buscando voltar ao equilíbrio.

Faz-se necessário rever os princípios e critérios norteadores do pensamento sistêmico, que a partir dos avanços da genética e da microbiologia, integram dimensões biológicas, cognitivas, e sociais, demonstrando que a vida em diferentes níveis está inextricavelmente interligada por redes complexas (Capra, 1996, 2002).

O pensamento sistêmico define primeiro a mudança de foco das partes para o todo, o que implica que um sistema deixa de existir ao ser "dissecado em elementos isolados" (Capra, 1996:46; Bertalanffy, 2010). Segundo, o mundo vivo constitui-se de sistemas dentro de sistemas, o que nos leva a considerar níveis diferentes de complexidade dos diferentes sistemas. Terceiro, que o todo não pode ser analisado "em termos das propriedades de suas partes" (Capra, 1996:47; Bertalanffy, 2010) como colocado pelo pensamento cartesiano.

Finalmente, o pensamento sistêmico aponta para "a percepção do mundo vivo como uma rede de relações" (Capra, 1996:47) além de suas partes em si. Este pensamento considera as relações que se estabelecem dentro do sistema como 
mais relevante que as partes em si. Ao considerar como mais importante estas relações, buscase entender os padrões de relacionamento entre as partes, ou seja, da rede que se estabelece em um dado sistema. Com isso, busca-se entender muito mais os fatores e vetores que influenciam as relações.

Ao adotar o conceito de redes dentro de redes, preocupa-se com o sistema vivo dentro de sistemas. Assim, um sistema vivo é parte de um todo maior. Sem um meio ambiente um sistema não existe (Maturana, Apud. CAPRA, 2002). É por intermédio das interações com o meio ambiente que os principais metabolismos são possíveis, permitindo que um sistema cresça e se reproduza.

Capra (1996) define "padrão de organização de um sistema vivo como a configuração das relações entre os componentes do sistema; a estrutura do sistema como a incorporação material desse padrão de organização; e o processo vital como o processo contínuo dessa incorporação" (CAPRA, 2002: 83).

À medida que sistemas sobrevivem, suas redes estão conectadas a outras redes, e por possuírem uma estrutura dissipativa que lhes permite estar em contínuo contato com o seu meio (e por este contato ser cognitivo) implica que ao interagir aprende-se e se reorganiza, ao mesmo tempo em que coloca nova informação no ambiente. Ao aplicar este conceito a sistemas sociais outros fatores e dimensões devem ser incorporados, não presentes em sistemas biológicos como regras de comportamento, valores, intenções, objetivos, crenças e estratégias.

Sistemas sociais, assim como, sistemas biológicos estabelecem redes de comunicações, que permitem trocas de informações entre organismos e seus meios. É por meio destas redes que se produz o conhecimento, tanto o conhecimen- to silencioso e interno, diretamente ligado a experiências, habilidades adquiridas e/ou desenvolvidas, criatividade e crenças; como o conhecimento explícito, ou seja, o que é externado por meio de linguagem verbal, eletrônica, impressa, entre outras (CAPRA, 2002).

Ao aplicar esses princípios à CPIC, Blumenschein (2004:116) conclui que o número de agentes atuantes na cadeia produtiva da indústria da construção, em conjunto com o número de fatores internos e externos que a influenciam, tornam-na naturalmente comparável aos sistemas vivos complexos, que sobrevive dentro de um ecossistema, ou seja, na economia e na sociedade.

A partir do momento que a CPIC é considerada análoga a um sistema vivo, pode-se identificar seu padrão, sua estrutura, seu processo e seu significado, de acordo com os critérios de caracterização de sistemas vivos colocados por Capra (1996, 2000).

\subsection{História Natural da Inovação}

A aplicação dos princípios da teoria de inovação e teoria dos sistemas no desenvolvimento e teste de metodologias de aceleração de mudança de paradigmas permitem avanços na estrutura de análises que visam o fortalecimento dos sistemas de aprendizados de organizações (BLUMENSCHEIN, 2004). Esses avanços levam a ampliar as lentes de análise focadas no conceito de inovação e na identificação dos vetores de influência que fortalecem o seu surgimento e evolução.

Johnson (2010) oferece uma abordagem que enfatiza a complexidade da interconexão dos vetores que influenciam o surgimento de inovações e ao apresentar sua analogia de banco de corais confirma a importância das redes colaborativas. 
A complexidade dos vetores de influência é apresentada em um esquema estruturado a partir de uma ampulheta que permite uma leitura ampliada desses vetores (figura 1). Na parte superior, à medida que se aproxima do centro a escala biológica se reduz. No centro há uma mudança de ambiente e escala: do ambiente natural para o ambiente cultural, mais amplo e complexo. Do centro para a parte inferior a escala se amplia novamente e é introduzida uma nova variável que é a capacidade de conexão e cooperação em rede.

Sob esta perspectiva, a capacidade de sobrevivência dos bancos de corais, não é resultado da capacidade de sobrevivência do mais forte, mas da capacidade de reinventar soluções a partir dos resultados das trocas e interações que permitem descobrir novas formas de sobrevivência. Esta é a explicação definitiva do paradoxo de Darwin: "os bancos de corais descobriram tantas formas de sobrevivência devido a forma como compartilham" (JOHNSON, 2010:245).

Ao aplicar esta analogia ao contexto de aprendizado das organizações o compartilhamento de recursos, expertises e responsabilidades

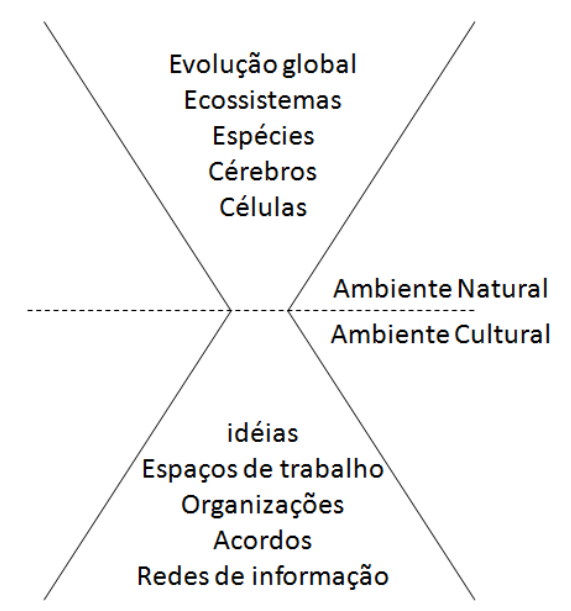

Figura 01: Níveis de escala dos ambientes natural e cultural na história da inovação. Adaptado de Johnson, 2010. pode ser considerado como um fator preponderante no processo de inovação.

\section{Aplicação dos princípios e a concepção do LACIS}

A aplicação dos princípios, identificados acima, no desenvolvimento de pesquisa aplicada no LACIS é resultado de uma trajetória iniciada na Universidade de Londres em 1988 e que recebeu fortes influências do Schumacher College em Devon (1991), e se fortaleceu no atendimento ao doutorado junto ao Centro de Desenvolvimento Sustentável da UnB (BLUMENSCHEIN, 2004).

O doutorado junto ao CDS/UnB permitiu avançar na pesquisa proposta. A amplitude do objeto de estudo, a complexidade da análise e a dificuldade para se obter dados exigiram uma combinação de diferentes métodos de pesquisa. Na busca desses caminhos foi necessário identificar os principais vetores de influência de mudanças industriais.

$\mathrm{Na}$ tentativa de confirmar vetores de influência aplicou-se o conceito de sistema nacional de aprendizado ao contexto da IC, visando obter um diagnóstico preliminar do potencial de aprendizado, portanto de mudanças. Posteriormente, trabalhou-se na montagem e na coordenação de projetos pilotos, que exercitaram a consolidação de princípios, critérios, informações e experiências, visando introduzir um paradigma tecnológico sustentável para o processo construtivo da CPIC. Os primeiros estudos de caso tiveram como objeto específico, a minimização dos impactos causados pelos resíduos sólidos oriundos da CPIC.

O primeiro piloto implantado e testado foi o Programa de Gestão de Materiais (PGM) ${ }^{1}$. Este Programa tem como objetivo central contribuir 
com a gestão dos materiais utilizados no processo construtivo, visando à minimização de impactos ambientais desde a extração de matérias-primas ao descarte de resíduos. Está composto de três subprogramas: Programa de Racionalização e Redução de Perdas; Programa de Análise do Ciclo de Vida dos Materiais; Programa de Gerenciamento de Resíduos Sólidos (BLUMENSCHEIN, 2004).

O PGM permitiu confirmar a hipótese de que para acelerar o processo de mudança da CPIC faz-se necessário integrar agentes que exercem o papel de coordenação na produção do ambiente construído (universidade, setor produtivo, setor público e terceiro setor); instrumentos (legais e econômicos); ações; políticas públicas; recursos, favorecendo a absorção de tecnologia e metodologia através do fortalecimento do seu sistema de aprendizado.

A busca pelo fortalecimento do sistema de aprendizado da CPIC, também fundamentou e estabeleceu diretrizes para aplicação e montagem de estudos de casos testados na prática. E o foco passou a ser no exercício de aceleração da disseminação de tecnologias sustentáveis.

Como resultado, desenvolveram-se métodos que norteiam, organizam e dirigem passos que são dados na prática para viabilizar a criação de redes que integram atores sociais capazes de fazerem mudanças relevantes e mais rápidas.

Os resultados dos programas e projetos desenvolvidos pelo LACIS confirmam que ao fortalecer os sistemas de inovação e aprendizado potencializa-se uma nova forma de conhecimento, que valoriza a maneira sustentável de materializar o ambiente construído, por cada agente envolvido na CPIC, compartilhando responsabilidades e recursos e integrando ações e instrumentos. Algumas lições aprendidas a partir de Blumenschein (2004) vem sendo ratificadas:
- O potencial das parcerias como meio de viabilizar o funcionamento de complexos sistemas sociais e organizacionais compondo redes colaborativas com objetivos específicos.

- A capacidade da rede de economizar aplicação de recursos e distribuir esforços para o fortalecimento da concretização de objetivos.

- A importância de consolidar significados comuns aos agentes da rede.

- A força dos instrumentos legais que podem pressionar a mudança de comportamento.

- A força que deve ser aplicada por grupos de clientes na decisão do produto a ser produzido e do seu processo de produção.

- O papel da mídia na disseminação e monitoramento de resultados.

- A importância de disponibilizar metodologias práticas para introdução de novos paradigmas viabilizando acelerar mudanças.

- O reconhecimento da dimensão políticoinstitucional como ponto critico da sustentabilidade.

A experiência do PGM permitiu a absorção de um aprendizado que passou a ser aplicado à gestão de resíduos sólidos urbanos e no teste de modelos de inclusão social e tecnológica com o objetivo de exercitar conceitos do metabolismo urbano regenerativo ${ }^{2}$. Entre as principais conclusões cita-se que o metabolismo urbano regenerativo é uma função da integração de agentes, ações e instrumentos: $\mathbf{M R U}=\mathbf{f}$ (integração de agentes + ações + instrumentos). Um princípio que passou a nortear a concepção e o desenvolvimento dos projetos de pesquisa aplicada do LACIS.

$* * *$ 


\section{Projetos e resultados}

Entre os principais projetos de pesquisa e respectivos resultados citam-se:

1. PGM - o Programa de Gestão de Materiais tem como objetivo central contribuir com a gestão dos materiais utilizados pelo processo construtivo, visando à minimização de impactos ambientais gerados desde a fase de extração de matéria prima até o descarte na forma de entulho. A concepção do PGM é resultado do trabalho em parceria do LACIS e a Comissão de Materiais (COMAT) do Sinduscon-DF, a partir do projeto de pesquisa de Doutorado da Professora Raquel Naves Blumenschein. O PGM foi concebido para ser implantado por meio de projetos pilotos, de acordo com a metodologia de elaboração, desenvolvimento, monitoramento e avaliação. Está composto de três programas: Programa de Gerenciamento de Resíduos Sólidos em Canteiros de Obras, PGRSC - PGM 01; Programa de Racionalização e Redução de Perdas PRRP - PGM 02 e Programa de Análise do Ciclo de Vida dos Materiais - PACVM - PGM 03. 2. PGM 01 - PGRSC -Programa de Gerenciamento de Resíduos Sólidos em Canteiros de Obras, PGRSC PGM 01 já teve início, e tem entre seus objetivos responder à Resolução 307 do CONAMA, no que se refere às responsabilidades dos geradores de resíduos sólidos em canteiros de obras, construção e demolição (RSCD). Está em fase de disseminação da metodologia consolidada por meio do PGM Piloto 1: Distrito Federal e Goiânia - GO, contando hoje com trinta e nove canteiros que participaram do Programa em Brasília e Goiânia; e em torno de 2100 colaboradores sensibilizados com relação à gestão de resíduos sólidos em canteiros de obras; 65 engenheiros e 200 encarregados treinados para atender a resolução 307 do CONAMA que dis- põe sobre a gestão de resíduos sólidos de construção.

3. PGM 02 - PRRP - O PROGRAMA DE RACIONALIZAÇÃO E REDUÇÃO DE PERDAS que tem como objetivo o desenvolvimento de um sistema de avaliação da racionalização do projeto, incluindo as fases de concepção e planejamento, gestão, planejamento de empreendimento e concepção e detalhamento do produto. O principal resultado foi o desenvolvimento de uma metodologia de avaliação do processo de projeto que permite o fortalecimento do sistema de aprendizado das empresas locais preparando-as para a absorção de tecnologias e outras metodologias que visem a racionalização e a redução de perdas. Participaram do desenvolvimento do sistema de avaliação três construtoras do DF. O PRRP está sendo desenvolvido em sua segunda fase em parceira com o Instituto de Arquitetos de Brasil - IAB/DF com o objetivo de testar e ampliar o sistema de avaliação proposto em processos de projeto coordenados por escritórios de arquitetura.

4. PGM 03 - PACVM - O Programa de Análise do Ciclo de Vida dos Materiais tem como objetivo elaborar, desenvolver e implantar metodologia que visa subsidiar a análise de ciclo de vida da cadeia de materiais de construção, a partir do envolvimento de indústrias do centro-oeste e agentes relevantes ao processo.

5. POGISAM - O Programa de Otimização da Gestão Integrada de Saneamento Ambiental visa testar e desenvolver metodologias que fortaleçam o processo de elaboração e implantação de políticas públicas que subsidiem o Município na elaboração do Plano de Saneamento Municipal e supram deficiências de municípios de pequeno porte na gestão sanitária municipal, integrando agentes, ações, instrumentos e recursos em todos os níveis. A concep- 
ção deste projeto forneceu subsídios para o desenvolvimento da metodologia utilizado no PROATOS.

6. PROATOS - O PROGRAMA DE INTEGRAÇÃO DE ATORES SOCIAIS para a GESTÃO DE RESÍDUOS SÓLIDOS URBANOS tem como objetivo propor a utilização de tecnologias de gestão inovadoras para diagnosticar a situação dos resíduos sólidos urbanos, visando trazer para comunidades uma oportunidade de integração baseada na formação de redes de compartilhamento de informações, conhecimento, ações e instrumentos, trabalhando a comunicação dos diversos atores, atraindo investimentos e dinamizando os mercados de materiais. O PROATOS permite ainda a identificação de problemas de fluxo de comunicação, de pontos de estrangulamento na cadeia de resíduos recicláveis, de espaços de diálogo e interação, possibilitando a elaboração de um modelo replicável de gestão e a criação de diversas oportunidades. $\mathrm{O}$ primeiro piloto do $\mathrm{PRO}-$ ATOS recebeu apoio financeiro da Fundação Banco do Brasil (FBB) e teve como objetivo principal contribuir para a Gestão Integrada de Resíduos Sólidos Urbanos (GIRSU) no Distrito Federal (DF), fortalecendo o fluxo de comunicação entre o GDF e a Centcoop, integrando agentes, ações e instrumentos e promovendo a geração de trabalho e renda por meio do adensamento da cadeia de recicláveis.

7. PRAS - PROGRAMA DE RESPONSABILIDADE AMBIENTAL E SOCIAL NACADEIA PRODUTIVA DA INDÚSTRIA DA CONSTRUÇÃO - O PRAS é um projeto do LACIS/FAU/CDS/UnB em parceria com a Comissão do Meio Ambiente do SINDUSCONDF e a FIBRA subsidiado pelo Programa de Apoio à Competitividade das Micro e Pequenas Empresas (PROCOMPI) proposto pelo Sis- tema CNI, Sebrae, FIBRA e Sebrae-DF e foi concebido para contribuir para a sustentabilidade da cadeia produtiva da indústria da construção (CPIC). Entre seus objetivos busca-se identificar a dimensão da responsabilidade social para o setor da construção e avaliar como as empresas se estruturam para lidar com atividades de responsabilidade ambiental e social (RAS). A primeira fase teve como produto um conjunto de critérios que compõem um padrão para implantação de política de Compra Ambiental e Socialmente Responsável. Este padrão foi validado na realização de um projeto piloto, com a participação de vinte empresas construtoras do DF e por consulta a organizações governamentais, não governamentais e especialistas e gerou o Guia de Compra Responsável para a indústria da construção em fase de publicação. A segunda fase tem como objetivo implantar piloto de Rotulagem em seis materiais produzidos no DF. A terceira fase tem como objetivo aplicar o conceito de responsabilidade ambiental e social no canteiro de obras. A quarta fase tem como objetivo desenvolver programa de capacitação e inclusão de presidiários e presidiárias como agentes ambientais treinados para trabalharem em canteiros de obra em processo de certificação.

8. LARRC - O Laboratório de Reciclagem de Resíduos da Construção tem como objetivo implantar usina de reciclagem de resíduos classe A na Faculdade do Gama/UnB desenvolvendo pesquisa de novos materiais e novas aplicações dos agregados reciclados. Está em fase de implantação.

9. OBSR3DF - Observatório da cadeia de resíduos sólidos recicláveis - O Observatório de Resíduos Sólidos Recicláveis do Distrito Federal foi desenvolvido pelo Lacis/FAU/CDS/FGAUnB com o apoio da Secretaria de Ciência e 
Tecnologia para Inclusão Social do Ministério de Ciência e Tecnologia. O Observatório tem como objetivo estruturar as informações sobre gestão de resíduos sólidos recicláveis, integrando-as em um local único, fortalecendo a gestão do conhecimento sobre o tema. O Observatório é um instrumento de gestão do conhecimento da gestão de resíduos recicláveis e reciclados incluindo a coleta de informações, tratamento, armazenamento, formatação em diversos formatos (gráficos, página de internet, mapas geográficos temáticos, relatórios tabulares, relatórios textuais, cruzamento de informações de diversas fontes, etc.), disseminação e uso ostensivo do máximo de informação pelo público interessado pelo tema.

10. OBSCI-CPIC - O Observatório do Conhecimento para Inovação da Cadeia Produtiva da Indústria da Construção é uma parceira entre o LACIS e a Câmara Brasileira da Indústria da Construção (CBIC) e tem como missão pesquisar, coletar, analisar, validar, processar, armazenar e disseminar dados de toda a natureza que tenham correlação com a geração e disseminação de conhecimento para a inovação da cadeia produtiva da indústria da construção. $O$ primeiro piloto está sendo desenvolvido e implantado no DF e deverá ser disseminado para outras localidades do país a partir da criação de uma rede a ser construída inicialmente com a participação de cinco estados federativos em cada região do país.

11. OBSERVE UnB - O Observatório: Conhecimento para Inovação UnB é uma iniciativa do Decanato de Pesquisa e Pós-graduação (DPP) em parceria com o Laboratório do Ambiente Construído Inclusão e Sustentabilidade, da Faculdade de Arquitetura e Urbanismo, Centro de Desenvolvimento Sustentável e Faculdade Unb Gama (LACIS/FAU/CDS/FGA-UnB).
É um ambiente georreferenciado para compartilhamento ostensivo de dados, informações e conhecimento produzidos pela Universidade de Brasília. No Observatório poderão ser encontradas as áreas e temas de pesquisa, os pesquisadores e respectivos departamentos, as respectivas pesquisas e resultados, bem como a rede de relacionamentos.

12. NUESP - O Núcleo de Estudo e Pesquisa da Edificação Penal visa o desenvolvimento da cadeia produtiva de edificações penais dentro de uma ampla perspectiva do sistema jurídicopenal no qual está inserida. Nesta perspectiva, o Nuesp busca essencialmente contribuir para a correta aplicação da pena (incluindo a qualidade do espaço da edificação penal - processos e produtos para a materialização deste espaço), o respeito da condição humana no sistema penal e a reinserção e inclusão sociais dos presos. 13. PISAC - O Parque de Inovação e Sustentabilidade tem como objetivo implantar um Parque de Inovação e Sustentabilidade no Campus da Faculdade do Gama (UnB) com foco no ambiente construído (processos e produtos) por meio de um consórcio de parceiros do setor público e privado do Reino Unido e do Brasil constituído pelas instituições: Building Research Establishment (BRE-Inglaterra); Câmara Brasileira da Indústria da Construção (CBIC) e Universidade de Brasília (UnB) por meio do LACIS que compartilham da mesma visão de desenvolver o futuro sustentável do Brasil por meio de inovação e padrões de sustentabilidade do ambiente construído.

Os resultados dos programas implantados pelo LACIS de 2004 a 2011 estão sumarizados no Quadro 01 e o balanço de projetos e programas elaborados, implantados e em processo de implantação estão apresentados no Quadro 02. 


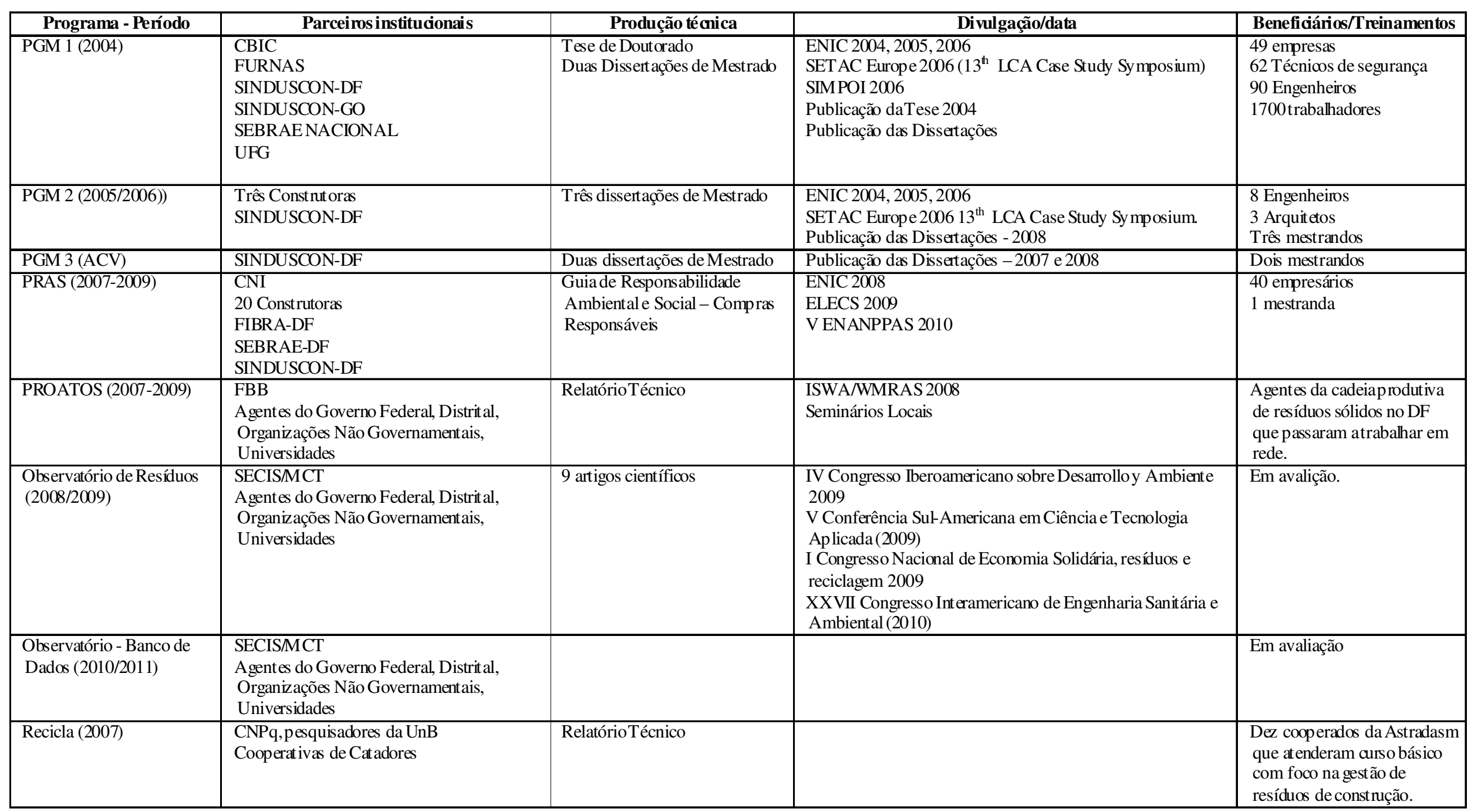


Quadro 02: Balanço dos projetos e programas do LACIS de 2004 a 2011

\begin{tabular}{|c|c|c|c|}
\hline Elaborados & Em process ode implantação & $\begin{array}{c}\text { Em } \\
\text { desenvol vimento }\end{array}$ & Concluídos \\
\hline Casa Legal (2007) & $\begin{array}{l}\text { Capacitação e Treinamento: } \\
\text { Sus tentabilidade em Canteiros de } \\
\text { Obras e Inclusão (2011) }\end{array}$ & $\begin{array}{l}\text { Banco de Dados do } \\
\text { Obs ervatório (2010 } \\
-2011)\end{array}$ & PGM 1 \\
\hline $\begin{array}{l}\text { Chamada Pública de Pesquis a } \\
\text { Científica (BNDES/FEP 02/2010) }\end{array}$ & NUESP (2011) & & PGM 2 \\
\hline $\begin{array}{l}\text { Compras Públicas Municipais } \\
\text { Responsáveis (2010) }\end{array}$ & $\begin{array}{l}\text { Observa UnB Observatório de } \\
\text { Pesquis a (2011) }\end{array}$ & & $\begin{array}{l}\text { PRAS - Fase } 1- \\
\text { Compra } \\
\text { Responsável }\end{array}$ \\
\hline $\begin{array}{l}\text { Inclusão de alunos de curso superior, } \\
\text { oriund os da rede pública, em projetos } \\
\text { de pesquisa aplicada (2010) }\end{array}$ & $\begin{array}{l}\text { Observatório do Conhecimento } \\
\text { para Inovação da CPIC (2011) }\end{array}$ & & PROATOS \\
\hline POGISAM (2006) & $\begin{array}{l}\text { Parque de Inovação e } \\
\text { Sustentabilidade (2011) }\end{array}$ & & \\
\hline $\begin{array}{l}\text { PRAS - Compras Responsáveis STJ } \\
(2009)\end{array}$ & $\begin{array}{l}\text { PRAS - Fase 2(2011) } \\
\text { Inclusão de pequenos Infratores }\end{array}$ & & \\
\hline PROATOS Ceilândia-DF (2006) & PROATOS - RIDE (2011) & & \\
\hline PROATOS São Sebastião (2007) & & & \\
\hline $\begin{array}{l}\text { PRONARC Programa Nacional de } \\
\text { Racionalização de Recursos na } \\
\text { Construção () (2007) }\end{array}$ & & & \\
\hline $\begin{array}{l}\text { Unidade de Reciclagem de Resíduos da } \\
\text { Construção - Prefeitura do Campus } \\
\text { Darcy Ribeiro (2007) }\end{array}$ & & & \\
\hline $\begin{array}{l}\text { Unidade de Reciclagem de Resíduos da } \\
\text { Construção - FGA (2009) }\end{array}$ & & & \\
\hline
\end{tabular}

\section{Considerações finais}

Os cinco anos de história do LACIS formam uma trajetória de aprendizado organizacional em que as lições aprendidas são importantes para desenhar os próximos passos.

O LACIS tem clareza de seu papel institucional, conta com uma equipe sinérgica, uma rede colaborativa de parceiros institucionais no governo federal, distrital, setor produtivo, acadêmico e terceiro setor que integram todos os programas e projetos desenvolvidos.

Ao longo dos cinco anos consolidaramse alguns princípios que norteiam a elaboração e desenvolvimento de pesquisa aplicada no LACIS:

1. Integrar agentes (setor público, privado, terceiro setor, academia), ações e instrumentos (legais, técnicos e sociais) para a geração de soluções;
2. Buscar soluções que contribuam com a manutenção dos principais processos ecológicos e os sistemas de suporte à vida;

3. Criar redes colaborativas para o compartilhamento de responsabilidades, tecnologias e conhecimento;

4. Contribuir para a inovação por meio do fortalecimento do sistema de aprendizado de agentes e organizações;

5. Promover a educação ambiental como vetor de mudança de paradigmas e contribuição aos processos regenerativos;

6. Promover a inclusão social por meio do desenvolvimento e compartilhamento de tecnologias sociais;

7. Contribuir para elaboração e implantação de políticas públicas;

8. Integrar ferramentas de gestão do conhecimento aos métodos de trabalho; 
9. Adotar padrões urbanos mais sustentáveis em planos, projetos e ações que protejam as capacidades regenerativas da Terra.

A sociedade vive um momento em que muitas oportunidades podem ser consideradas, devido ao momento político atual que favorece a consolidação de parcerias locais, bem como as demandas crescentes por responsabilidade ambiental e social pelos agentes públicos e privados, Municípios, empresas, cidadãos, políticos, cadeias produtivas, agentes financeiros e academia.

\section{Bibliografia}

BERTALANFFY, L. V. Teoria Geral dos Sistemas. Editora Vozes Ltda., Petrópolis, 2010.

BLUMENSCHEIN, R. N. A Sustentabilidade na Cadeia Produtiva da Indústria da Construção, 252p., UnB, CDS, Doutorado em Política e Gestão Ambiental, Brasília, 2004.

BLUMENSCHEIN, R. Three studies of innovation in the construction industry. 1989. 66f. Dissertação (Mestrado em Economia e Administração Aplicadas à Indústria da Construção). Bartlett School of Architecture and Planning. University College London.

CAPRA, F. As conexões ocultas. São Paulo: Editora Cultrix. 2002. 296p.

CAPRA, F.A. A teia da vida. São Paulo. Cultrix. 1996. 256p.

DOSI, G. et al. Technical change and economic theory. Great Britain: Pinter Publishers, 1988, $641 p$.

FREEMAN, C. The economics of technical change. Cambridge Journal of Economics. England, v. 18, p463-514, 1994.

FREEMAN, C. The national system of innovation in historical perspective. Cambridge Journal of Economics. England, v. 19, n.1, p. 5-22, feb 1995.

JOHNSON, S. Where Good Ideas Come From: the Natural History of Innovation; Penguim Books, Londres, 2010.

LACIS. Planejamento Estratégico. FAU/CDS/ FGA-UnB, Brasília, 2011.

LEGISLATIVE ASSEMBLY OF QUEENSLAND. Nature Conservation Act. Act No 20. Queensland 1992. Reprinted em june 2010. LEMOS, C. Inovação na era do conhecimento. Parcerias estratégicas, Brasília, n.8, p. 157179, maio, 2000.

LYLE, J. T. Regenerative Design for Sustainable Development. New York: John Wiley \& Sons, 1994. cap. 10, p. 281-305.

SCHUMPETER, J.A. The theory of economic development. Cambridge (Mass): Harvard University Press, 1949.

SCHUMPETER, J.A. Business cycles. Vol.I. New York: McGraw-Hill Book Company Inc., 1939.

SILVA, A. B. \& REBELO, L.M.B. A emergência do pensamento complexo nas organizações. Revista de Administração Pública, Rio de Janeiro, v. 37, 777-796, jul/ago 2003.

VIOTTI, E.B. Passive and active national learning systems. 1997. 311f. Tese (Doutorado em Filosofia). The Graduate Faculty of Political and Social Science of the New School for Social Research, EUA.

\section{Notas}

${ }^{1}$ Cadeia produtiva como o "conjunto de atividades que se articulam progressivamente desde os insumos básicos até o produto final, incluindo distribuição e comercialização, constituindo-se em elos de uma corrente" (MDIC, 2002:03). Ao aplicar este conceito ao universo da indústria da construção (IC), pode-se afirmar que esta indústria é composta por três gru- 
pos industriais básicos (i) indústria que produz materiais, insumos e componentes (ou indústria de suprimentos); (ii) indústria que produz edificações e obras de engenharia pesada (ou indústria de processos); indústria considerada auxiliar. Esta última no âmbito do LACIS/FAU/ CDS/FGA vem sendo tratada como cadeia do conhecimento e inteligência (Blumenschein, 2004:44)

${ }^{2}$ Segundo Blumenschein (2004) "um paradigma tecnológico estabelece-se ao longo de um processo de acumulação de conhecimentos e "maneiras de se fazer e ou produzir". Dosi (1988) afirma que paradigma tecnológico "define contextualmente as necessidades a serem preenchidas, os princípios científicos utilizados para a tarefa e o material tecnológico a ser usado" (DOSI, 1988, pg.224).

${ }^{1}$ Resultado de uma parceria entre Universidade de Brasília, Sinduscon/DF, Sinduscon/GO, Câmara Brasileira da Indústria da Construção, Prefeitura de Goiânia, Furnas Centrais Elétricas S.A, Universidade Federal de Goiás.

${ }^{1} \mathrm{O}$ conceito de metabolismo urbano regenerativo fundamenta-se na busca de soluções que inserem na vida das cidades fluxos circulares (transformadores de maneira equilibrada) de energia, produtos, pessoas, água, resíduos, poluentes, entre outros. Uma analogia em desenvolvimento com base em Lyle (1994), World Future Council www.futurepolicy.org, BRE (England - www.bre.co.uk), entre outros. 
